\title{
EXPERIENCE RATING SCHEMES FOR FLEETS OF VEHICLES*
}

\author{
BY \\ Denise Desjardins ${ }^{1}$, Georges Dionne ${ }^{1,2}$, Jean Pinquet $^{3}$
}

\begin{abstract}
This paper proposes bonus-malus systems for fleets of vehicles, by using the individual characteristics of both the vehicles and the carriers. Bonus-malus coefficients are computed from the history of claims or from the history of safety offences of the carriers and the drivers. The empirical results are derived from a data set obtained from the Société de l'Assurance Automobile du Québec, the public insurer for bodily injuries and the regulator of road safety.
\end{abstract}

\section{KEYWORDS}

Stratified portfolios, credibility, vehicle, fleet, accidents, safety offences.

\section{INTRODUCTION}

This paper stems from a study carried out for the Société de l'Assurance Automobile du Québec, later referred as the SAAQ (see also Dionne, Desjardins, Pinquet (1999, 2000a)). Its objective is to provide Bonus-Malus Systems (later referred to as BMS) for fleets of vehicles from the history of claims or from that of safety offences.

Fleets of vehicles are owned by firms, which are commercial motor carriers in the SAAQ portfolio. A portfolio of insurance contracts subscribed by firms has a stratified structure, and the size of the stratum (the set of policies held by

\footnotetext{
* The paper benefited from useful comments of two anonymous referees. This research was funded by the programme de recherche universitaire en sécurité routière of the Ministère des Transports $\mathrm{du}$ Québec (MTQ) and the Société de l'Assurance Automobile du Québec. The authors also acknowledge financial support from the Fédération Française des Sociétés d'Assurances (FFSA) and the FCAR in Quebec. They remain responsible for the errors, if any. A first version was presented at two research meetings of the FFSA and at the Risk Theory Seminar of the American Risk and Insurance Association.

1 Centre for Research on Transportation, University of Montreal.

2 Risk Management Chair, HEC-Montreal.

3 (Corresponding Author) University Paris X.
} 
a given firm) is a key variable in risk analysis. The propensity to self-insurance increases with the size of the stratum. Insurance contracts for fleets of vehicles often use stop-loss risk sharing schemes (see Marie-Jeanne (1994) for their properties as a function of the fleet size, and Teugels, Sundt (1991) for experience rating schemes on the aggregate loss). These rating structures are designed for large fleets, which is not the case on average for the portfolio analyzed in this article. Notice that, in general, fleet insurance business is offered mostly for fleets with little or medium size.

In our data set, the characteristics of each fleet are recorded by the SAAQ in real-time (see Section 2), and the tariff structures proposed in this article use the individual characteristics of both the vehicles and the carriers. The history of a vehicle should have a greater ability to predict the risk level of this vehicle than that of the other vehicles in the fleet. The basic issue in the statistical analysis of the portfolio is the assessment of these predictive abilities. Information on the drivers is not available in the data set, so a new vehicle can only be related to the fleet to which it belongs. Bonus-malus coefficients for the next period will then depend on an expected turnover for the vehicles of the fleet. Since the insurance premium is paid at the firm level, the bonusmalus coefficients computed in the paper depend on the history of claims or safety violations at the fleet level. However, an experience rating scheme using full information on the claims history is designed in Section 3.5.

The experience rating schemes are based on models with hierarchical random effects (see Jewell (1975)). Two types of BMS are analyzed. BMS designed from the number of claims are presented in Section 3, and another one obtained from the history of safety offences is given in Section 4 . We explain the number of claims for bodily injuries. Bonus-malus coefficients are obtained from vehicle-specific and fleet-specific credibilities. They take into account an expected turnover for the vehicles within the fleets.

Compensations for bodily injuries are performed in Quebec within a pure no-fault framework (Devlin (1992); Boyer and Dionne (1987)), so it is difficult to use the history of claims in the rating structure, because standard BMS always have a "crime and punishment" flavour. Since 1992, the history of safety offences is used in the tariff structure of the SAAQ for pleasure vehicles (see Dionne and Vanasse (1997a) and Dionne, Maurice and Pinquet (2000b) for a related study).

The BMS designed in Section 3 is consistent with respect to the fleet-specific components, which is not the case when claims are replaced by safety offences as in Section 4. However, the BMS based on safety offences outperforms the one based on accidents after a year of experience with our data. The explanation of this somewhat surprising finding is the following. The frequency of offences is fourteen times higher than that of claims with bodily injuries. Even if the BMS based on safety offences is less efficient than the one based on accidents in the long run, the former system is closer to its limit in the short run, due to the higher frequency of safety offences.

A short conclusion summarizes the main results and proposes some extensions to the models presented in this article. 


\section{ECONOMIC ENVIRONMENT AND DATA SET}

Let us precise first the context of the study. The Province of Quebec introduced a new Automobile Insurance Act in March, 1978 to govern accident compensations. The Government had two goals in mind in tabling this legislation - to provide a rapid and reliable method for compensating all victims of bodily injuries, and to ensure better control of the cost of car repairs and faster compensation for property damage.

Fault has been entirely eliminated for bodily injuries. Compensation is provided by a compulsory and universal public plan. This plan is administered by a public corporation, the SAAQ. There is a maximum indemnity (which was estimated to compensate the total loss of income of 85 per cent of the population in 1978) for disability and death benefits. The indemnities for bodily injuries are in lieu of all rights to sue for bodily injuries or death, and no action is admitted before any court of justice.

The pricing procedure is very simple. The main sources of financing are from drivers' permits and automobile registration fees. Weight and type of vehicle driven are taken into consideration for vehicles other than pleasure vehicles. Past driving experience is taken into account since 1992 by using demerit points of the drivers.

So the SAAQ is a state insurer which provides motor insurance for bodily injuries in a monopolistic situation. As a state company, the SAAQ is also involved in road safety regulation. Consequently, it has a direct access to the information on individual safety offences. It was decided in 1992 to use such information for the pricing of private cars insurance. Besides their ability of screening risks, experience rating schemes provide incentives to careful driving. Indeed, the frequency of claims decreased by at least five per cent since the new regulation (see Dionne, Maurice and Pinquet (2000b) for more details).

The SAAQ also provides insurance for bodily injuries for fleets of vehicles. This insurance is also compulsory. Information is brought in real time for each vehicle, a situation which is not often encountered in this market. In order to create road safety incentives, the introduction of an experience rating scheme (as well as an a priori rating structure) is under consideration, which motivated the present study. This type of insurance rating would be easy to implement for the SAAQ since it has a direct access to all the necessary data.

Since January 1991, the SAAQ has been mandated to verify that commercial vehicles respect the laws and regulations governing, for example, the vehicle load and size limits, etc. In addition, the SAAQ was also given the mandate to verify the mechanical conformity of the vehicles.

In our working sample, the vehicles were observed during the years 1995 and 1996. The duration of observation of a vehicle is the validity duration of its licence plate. The weight of the vehicles has to be greater than $3,000 \mathrm{kgs}$, hence fleets of cars do not belong to this sample. The portfolio contains 50,746 fleets and 124,629 vehicles, and fleets are of small size on average. The size of the fleet is measured in vehicle-years, which is the sum of the validity durations. The other fleet-specific rating factors are the age of the firm and its 
activity sector. The vehicle-specific rating factors are the weight, the type of use, the type of fuel, the number of cylinders and the number of axles.

The initial file is the file of all registered motor carriers as of July 23, 1997. To be in that file a motor carrier must own or lease (long term) one or more vehicles.

We matched the information concerning the vehicles and the firms with the characteristics of safety violations committed at the carrier or at the vehicle level. The characteristics concerning mechanical conformity of the vehicles which had a recent mechanical check-up were linked to the data set already obtained.

The unit of observation in the working sample is a vehicle with at least one day with a valid license plate in 1996. In considering the safety offences committed in 1995 in the analyses, 24,581 trucks with no day with a valid license plate in 1995 have been dropped from the data set.

\section{BONUS-MALUS SYSTEMS FROM THE NUMBER OF CLAIMS}

\subsection{Bonus-malus coefficients as functions of the size of the fleet: Two limit examples}

On a stratified portfolio, fixed and random effects introduced to design an optimal BMS must have a hierarchical structure (Jewell (1975)). The risk distribution of each vehicle includes then a vehicle-specific effect and a fleet-specific effect. Let us compute bonus-malus coefficients in two limit situations:

- Only the vehicle-specific effect is retained. The history of a vehicle cannot be used to predict the risk levels of the other vehicles in the fleet. If all the vehicles have the same a priori frequency risk, the credibility computed at the fleet level is the one given to each vehicle. As the variance of the ratio between the number of claims and the frequency premium decreases towards 0 when the size of the fleet goes to infinity, the same result holds for the variance of the bonus-malus coefficient.

- Only the fleet-specific effect is included in the number of claims distribution. Denote $m$ as the number of vehicles in a given fleet, $n_{i}$ as the number of claims reported by the vehicle $i$ and $\lambda$ as the a priori frequency risk for all the vehicles. We then have

$$
N_{i} \sim P(\lambda u)(\forall i=1, \ldots, m) \Rightarrow \sum_{i=1}^{m} N_{i} \sim P(m \lambda u),
$$

if the $N_{i}$ are independent in the fixed effects model (the fixed effect common to the vehicles in the fleet is denoted as $u$ ). If we write $E(U)=1 ; V(U)=\sigma^{2}$ in the random effects model, the credibility granted to the fleet in the prediction is equal to

$$
a=\frac{m \widehat{\hat{\lambda}} \widehat{\sigma^{2}}}{1+m \widehat{\lambda} \widehat{\sigma^{2}}}
$$


This credibility increases towards one when the size $m$ goes to infinity, and the bonus-malus coefficient converges towards the fleet-specific fixed effect $u$. The variance of the bonus-malus coefficient increases with the size of the fleet in the random effects model.

If the two random effects are included in a hierarchical model, the credibility granted to the history of the fleet will increase with its size if the estimated variance of the fleet-specific random effect is greater than zero. On the other hand, the variance of the bonus-malus coefficients is not a monotonic function of the size of the fleets. The increase of risk revelation with the size of the fleet is balanced by risk compensation between the vehicles.

\subsection{Estimation of a model with random effects on a stratified portfolio}

The hierarchical nature of the portfolio is taken into account by a double indexation. The fleets are indexed by $f=1, \ldots, F$, and the vehicles are indexed by $i=1, \ldots, m_{f}$, where $m_{f}$ is the size of the fleet $f$. If $N_{f i}$ is the number of claims reported by the vehicle $i$ in the fleet $f$, we write

$$
N_{f i} \sim P\left(\lambda_{f i} u_{f i}\right) ; f=1, \ldots, F ; i=1, \ldots, m_{f}
$$

in the fixed effects model. The number of claims $N_{f i}$ follows a Poisson distribution in the fixed effects model. The parameter $\lambda_{f i}$ is a function of rating factors observed at the fleet level or at the vehicle level. The fixed effect $u_{f i}$ represents the residual heterogeneity in the number of claims distribution. We distinguish firm-specific and vehicle specific effects in the regression and heterogeneity components, and write

$$
\lambda_{f i}=d_{f i} \exp \left(x_{f} \gamma+z_{f i} \delta\right) ; u_{f i}=r_{f} s_{f i} .
$$

The parameter $\lambda_{f i}$ is proportional to the duration of observation of the vehicle $d_{f i}$. The line-vectors $x_{f}$ and $z_{f i}$ are the regression components connected to the fleet and to the vehicle. The related parameters are represented by the column-vectors $\gamma$ and $\delta$. The fixed effect $u_{f i}$ splits into a fleet-specific effect $r_{f}$ and a vehicle-specific effect $s_{f i}$. Vehicle-specific heterogeneity components could reflect the behaviour of the drivers, if a given vehicle is used by few drivers. This heterogeneity component can also reflect hidden features which are only related to the vehicle. You might think of annual mileage, which depends on the missions assigned to a given truck, but not on the drivers. The behaviour of the firms will influence the fleet-specific heterogeneity components. Fleet owners may obey (or not) to safety rules related to the mechanical check-up of vehicles, bulk trucking regulation, driving and work hour rules, etc. The financial structure of the carrier (which is not recorded by the SAAQ) probably influences safety activities, and hence the risk level. Economic and empirical results on the relationship between the financial structure of air carriers and safety are given by Dionne et al. (1997b). 
The preceding distributions hold for real individuals, and the variables $\left(N_{f i}\right)_{f=1, \ldots, F ; i=1, \ldots, m_{f}}$ are supposed to be independent in the fixed effects model. This is the usual assumption in actuarial models (observed contagion on risk variables is supposed to be only apparent). The random effects $\left(R_{f}\right)_{f=1, \ldots, F}$ and $\left(S_{f i}\right)_{f=1, \ldots, F ; i=1, \ldots, m_{f}}$ are i.i.d. in each family and mutually independent. Distributions in the model with random effects are mixtures of Poisson distributions, and they refer to generic individuals, who represent a class of real individuals with the same observable characteristics (see Pinquet (2000) for instance). The independence between the $\left(R_{f}\right)_{f=1, \ldots, F}$ and $\left(S_{f i}\right)_{f=1, \ldots, F ; i=1, \ldots, m_{f}}$ can be assumed without loss of generality since the decomposition $U_{f i}=R_{f} S_{f i}$ is not unique. The random effect $S_{f i}$ reflects a residual heterogeneity in the risk distribution of the vehicle. If $R$ and $S$ are random variables with these distributions, we suppose that

$$
E(R)=E(S)=1 ; V(R)=V_{R R} ; V(S)=V_{S S} .
$$

Within a semiparametric approach, the distributions on the random effects will only be specified by the variances. If $U=R S$, we have

$$
E(U)=E(R) E(S)=1 ; V(U)=V_{U U}=E\left(R^{2}\right) E\left(S^{2}\right)-1=V_{R R}+V_{S S}+V_{R R} V_{S S} .
$$

With the total variance and covariance formula and the independence assumed in the model with fixed effects, we obtain

$$
\begin{gathered}
V\left(N_{f i}\right)=\lambda_{f i}+\lambda_{f i}^{2} V\left(U_{f i}\right)=\lambda_{f i}+\lambda_{f i}^{2} V_{U U} ; \\
\operatorname{Cov}\left(N_{f i}, N_{f i}\right)=\lambda_{f i} \lambda_{f t} \operatorname{Cov}\left(U_{f i}, U_{f t}\right)=\lambda_{f i} \lambda_{f i} V_{R R}\left(i \neq i^{\prime}\right)
\end{gathered}
$$

in the random effects model. As the size of the portfolio is large, we will use a frequentist approach, and will describe the data by consistent estimators.

The a priori rating model is a Poisson model without fixed or random effects, i.e. $N_{f i} \sim P\left(\lambda_{f i}\right) \forall f, i$. Let $\widehat{\lambda_{f i}}=d_{f i} \exp \left(x_{f} \widehat{\gamma}+z_{f i} \widehat{\delta}\right)$ be the frequency premium computed in the a priori rating model, where $\widehat{\gamma}$ and $\widehat{d}$ are the maximum likelihood estimators. The likelihood equations in this model are

$$
\sum_{f, i}\left(n_{f i}-\widehat{\lambda_{f i}}\right)^{t} x_{f}=0 ; \sum_{f, i}\left(n_{f i}-\widehat{\lambda_{f i}}\right)^{t} z_{f i}=0
$$

They reflect an orthogonality relationship between number-residuals and the regression components. Since $E\left(N_{f i}\right)=\lambda_{f i} E(U)=\lambda_{f i}$ in the model with random effects, the m.l.e. in the Poisson model without fixed and random effects are consistent estimators of the corresponding parameters in the model with random effects (see Gouriéroux et al. (1984)). Hence, a frequency premium computed for an individual in the a priori rating model converges towards the frequency risk of the related generic individual in the model with random effects. 
From the moments computed in (2), we obtain the following limits

$$
\begin{aligned}
& \widehat{V_{R R}}=\frac{\sum_{f} \sum_{1 \leq i, i^{\prime} \leq m_{f} ; i \neq i^{\prime}}\left(n_{f i}-\widehat{\lambda_{f i}}\right)\left(n_{f i^{\prime}}-\widehat{\lambda_{f i^{\prime}}}\right)}{\sum_{f} \sum_{1 \leq i, i^{\prime} \leq m_{f} ; i \neq i^{\prime}} \widehat{\lambda_{f i} \widehat{\lambda_{f i^{\prime}}}}} \rightarrow V_{R R} \\
& \widehat{V_{U U}}=\frac{\sum_{f, i}\left[\left(n_{f, i}-\widehat{\lambda_{f i}}\right)^{2}-n_{f i}\right]}{\sum_{f, i} \widehat{\lambda_{f i}^{2}}} \rightarrow V_{U U} \text {. }
\end{aligned}
$$

Thus consistent estimators of $V(U)$ and $V(R)$ are obtained from the estimators derived in the a priori model. Since $V_{U U}=V_{R R}+V_{S S}+V_{R R} V_{S S}$,

$$
\widehat{V_{S S}}=\frac{\widehat{V_{U U}}-\widehat{V_{R R}}}{1+\widehat{V_{R R}}}
$$

is a consistent estimator of $V_{S S}$.

Let us interpret these results. The estimator $\widehat{V_{R R}}$ assesses observed contagion between the claims histories connected to different vehicles within the same fleet. If $\widehat{V_{R R}}$ is greater than zero, the positive observed contagion means that the history of a vehicle can reveal hidden features in the risk distributions of every vehicle in the same fleet. The numerator of the ratio which defines the estimator $\widehat{V_{R R}}$ is easily derived from

$$
\sum_{f} \sum_{1 \leq i, i^{\prime} \leq m_{f} ; i \neq i^{\prime}}\left(n_{f i}-\widehat{\lambda_{f i}}\right)\left(n_{f i^{\prime}}-\widehat{\lambda_{f i^{\prime}}}\right)=\sum_{f}\left(n_{f}-\widehat{\lambda_{f}}\right)^{2}-\sum_{f, i}\left(n_{f i}-\widehat{\lambda_{f i}}\right)^{2},
$$

if we write $\sum_{1 \leq i \leq m_{f}} n_{f}, \widehat{\lambda_{f}}=\sum_{1 \leq i \leq m_{f}} \widehat{\lambda_{f i}}$. We then have

$$
\begin{aligned}
& \widehat{V_{S S}}>0 \Leftrightarrow \widehat{V_{U U}}>\widehat{V_{R R}} \\
& \Leftrightarrow \frac{\sum_{f, i}\left[\left(n_{f, i}-\widehat{\lambda_{f i}}\right)^{2}-n_{f i}\right]}{\sum_{f, i}{\widehat{\lambda_{f i}}}^{2}}>\frac{\sum_{f} \sum_{1 \leq i, i^{\prime} \leq m_{f} ; i \neq i^{\prime}}\left(n_{f i}-\widehat{\lambda_{f i}}\right)\left(n_{f i^{\prime}}-\widehat{\lambda_{f i^{\prime}}}\right)}{\sum_{f} \sum_{1 \leq i, i^{\prime} \leq m_{f} ; i \neq i^{\prime}} \widehat{\lambda_{f i}} \widehat{\lambda_{f f}}} \\
& \Leftrightarrow \frac{\sum_{f, i}\left[\left(n_{f, i}-\widehat{\lambda_{f i}}\right)^{2}-n_{f i}\right]}{\sum_{f, i}{\widehat{\lambda_{f i}}}^{2}}>\frac{\sum_{f}\left[\left(n_{f}-{\widehat{\lambda_{f}}}^{2}-n_{f}\right]\right.}{\sum_{f}{\widehat{\lambda_{f}}}^{2}}
\end{aligned}
$$

The estimated variance of the vehicle-specific random effect is greater than zero if the relative overdispersion derived at the vehicle level is greater than its counterpart computed at the fleet level. 
These moment-based estimators are unconstrained (i.e. estimated variances are not bound to be positive). Suppose for instance that $\widehat{V_{R R}}<0$ on a sample. Such an estimation would be related to a null estimator of $V_{R R}$ within a constrained approach (for instance m.l.e., which is costly to perform if the likelihood does not admit a closed form). In this case, the fleet-specific random effect must be abandoned whatever is the estimation strategy. Hence, the unconstrained nature of the estimators retained in the paper is not a drawback.

These estimators are consistent and asymptotically normal in the model with random effects. Their asymptotic variance can be reduced if weights related to overdispersion are introduced in the regression (see Liang, Zeger (1986)).

Let us precise this point. Denote the parameters of the a priori rating model and of the mixing distribution as

$$
\eta=\left(\begin{array}{l}
\gamma \\
\delta
\end{array}\right) ; \theta=\left(\begin{array}{l}
V_{R R} \\
V_{S S}
\end{array}\right) \text {. }
$$

If we stack the numbers of claims reported on a given fleet in a vector $s n_{f}=$ $v e c_{1 \leq i \leq m_{f}}\left(n_{f i}\right)$ the m.l.e. of the Poisson model (3) can be expressed as the solution in $\eta$ of the equation

$$
\sum_{f}^{2}\left(\frac{\partial}{\partial \eta} E\left(S N_{f} \mid \eta\right)\right)\left[V\left(S N_{f} \mid \eta\right)\right]^{-1}\left(s n_{f}-E\left(S N_{f} \mid \eta\right)\right)=0
$$

where the moments are computed in the Poisson model without fixed or random effects. Let $E\left(S N_{f} \mid \eta, \theta\right)$ and $V\left(S N_{f} \mid \eta, \theta\right)$ be the expectation and variance derived in the random effects model (we have $\left.E\left(S N_{f} \mid \eta, \theta\right)=E\left(S N_{f} \mid \eta\right) \forall \eta, \theta\right)$. The moment-based estimators of $V_{R R}$ and $V_{S S}$ given in this section from the regression provide a function $\eta \rightarrow \widehat{\theta}(\eta)$. A "generalized estimating equation" includes the estimated moments of the random effects in equation (6). The corresponding estimator is the solution in $\hat{\eta}$ of equation (6), where $V\left(S N_{f} \mid \eta\right)$ is replaced by $V\left(S N_{f} \mid \eta, \widehat{\theta}(\eta)\right)$. This estimator $\eta$ can be shown to have optimal properties in terms of asymptotic variance. Then a new estimator $\widehat{\theta}(\hat{\eta})$ is obtained for the parameters of the mixing distribution. The random effects model retained in this section is usually referred to as an "exchangeable correlations" model.

\subsection{Linear credibility predictors}

In this section, we compute linear credibility predictors (Bühlmann (1967)) for each vehicle. They are derived from the history of claims observed at the fleet level, whereas the credibility coefficient depends on the vehicle. Let $i_{0}$ be a vehicle which belongs to the fleet $f_{0}$. The portfolio is observed during one period, and a bonus-malus coefficient is computed for the next one. In order to allow for a turnover in the portfolio, this vehicle may appear at the second period. Predictors are obtained separately for each fleet, and the fleet index is suppressed in order to simplify the notations. The fleet is supposed to contain $m$ vehicles during the first period. 
The bonus-malus coefficient for the vehicle $i_{0}$ is supposed to depend only on the number of claims reported on the whole fleet. It is written as $\widehat{a}_{i_{0}}+$ $\widehat{b}_{i_{0}}\left(\sum_{i=1}^{m} n_{i}\right)$, with

$$
\left(\widehat{a}_{i_{0}}, \widehat{b}_{i_{0}}\right)=\arg \min _{a, b} \widehat{E}\left[\left(U_{i_{0}}-a-b\left(\sum_{i=1}^{m} N_{i}\right)\right)^{2}\right] .
$$

The estimated expectation is derived in the random effects model. Notice that no specific weight is given to the history of the vehicle. As $E\left(U_{i_{0}}\right)=1$, we have

$$
\widehat{a}_{i_{0}}+\widehat{b}_{i_{0}}\left(\sum_{i=1}^{m} n_{i}\right)=1+\widehat{b}_{i_{0}}\left(\sum_{i=1}^{m}\left(n_{i}-\widehat{\lambda}_{i}\right)\right)=\left(1-\operatorname{cred}_{i_{0}}\right)+\operatorname{cred}_{i_{0}} \frac{\sum_{i=1}^{m} n_{i}}{\sum_{i=1}^{m} \widehat{\lambda}_{i}}
$$

with

$$
\operatorname{cred}_{i_{0}}=\widehat{b}_{i_{0}}\left(\sum_{i=1}^{m} \widehat{\lambda_{i}}\right)=\frac{\widehat{\operatorname{Cov}}\left(U_{i_{0}}, \sum_{i=1}^{m} N_{i}\right)}{\widehat{V}\left(\sum_{i=1}^{m} N_{i}\right)}\left(\sum_{i=1}^{m} \widehat{\lambda_{i}}\right) .
$$

Consistent estimators for the individual moments are

$$
\begin{aligned}
& \widehat{\operatorname{Cov}}\left(U_{i_{0}}, N_{i}\right)=\widehat{\lambda_{i}} \widehat{\operatorname{Cov}}\left(U_{i_{0}}, U_{i}\right)=\left\{\begin{array}{l}
\lambda_{i} \widehat{V_{R R}}\left(i_{0} \neq i\right) \\
\lambda_{i_{0}} \widehat{V_{U U}}\left(i_{0}=i\right)
\end{array} ;\right. \\
& \widehat{V}\left(N_{i}\right)=\widehat{\lambda_{i}}+\widehat{\lambda_{i}}{ }^{2} \widehat{V_{U U}} ; \widehat{\operatorname{Cov}}\left(N_{i}, N_{i^{\prime}}\right)=\widehat{\lambda_{i}} \widehat{\lambda_{i^{\prime}}} \widehat{V_{R R}\left(i \neq i^{\prime}\right),}
\end{aligned}
$$

with the estimators obtained in the preceding section. In the computation of the credibility coefficient, two situations may happen:

- Either the vehicle was not observed during the first period, which means that it joined the fleet during the forecast period $\left(i_{0} \neq i \forall i=1, \ldots, m\right)$. From the estimations obtained in (7), we have

$$
\operatorname{cred}_{i_{0}}=a=\frac{\widehat{V_{R R}}\left(\sum_{i=1}^{m} \widehat{\lambda_{i}}\right)}{\left.1+\left(\widehat{V_{R R}}\left(\sum_{i=1}^{m} \widehat{\lambda_{i}}\right)\right)+\left(\widehat{\left(V_{U U}\right.}-\widehat{V_{R R}}\right) \frac{\sum_{i=1}^{m} \widehat{\lambda}_{i}^{2}}{\sum_{i=1}^{m} \widehat{\lambda_{i}}}\right)}
$$

This fleet-specific credibility coefficient roughly increases with the estimated variance of the fleet-specific random effect and with the frequency-premium computed at the fleet level.

- Or the vehicle was observed during the first period $\left(1 \leq i_{0} \leq m\right)$. Then

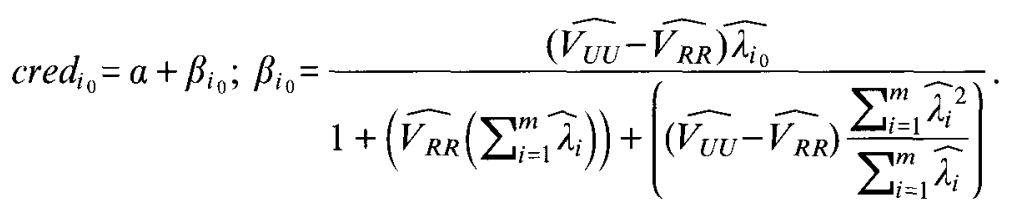


The credibility coefficient is the sum of the fleet-specific coefficient and of a vehicle-specific coefficient. It can be computed only if the estimated variance of the vehicle-specific effect $\widehat{V_{S S}}$ is greater than zero (which amounts to $\widehat{V_{U U}}>$ $\widehat{V_{R R}}$ from (5)), a condition fulfilled in our data. This coefficient is the bonus granted to the firm if no claim is recorded on its vehicles.

Fleets are open in most cases, which means that an endorsement is not brought to the insurance policy after each arrival or departure of a vehicle in the fleet. In this context, bonus-malus coefficients computed at the vehicle level may appear unrealistic. If $\rho$ is the expected turnover for the vehicles of the fleet, a credibility equal to $a+((1-\rho) \bar{\beta})$ can be retained at the fleet level, where $\bar{\beta}$ is the average of the $\beta_{i}$.

\subsection{Empirical results}

Table 1 presents the results of a Poisson model which explains the number of claims reported in 1996 by regression components derived from rating factors. The only continuous rating factor is the age of the firm. We observe that the frequency of claims decreases - ceteris paribus - by $3.4 \%$ with a supplementary year of age. The other rating factors have a finite number of categories.

In Table 1, the vehicles are weighted by the risk exposure measured by the number of days the vehicle is authorized to circulate. The estimated exponential of the coefficients (written in a multiplicative way) related to the different levels of each rating factor are averaged to one (column ST. COFF., for standardized coefficient). Two advantages are obtained.

- The coefficients do not depend on the category that must be omitted in the regression for each rating factor in order to avoid colinearity. This is due to the fact that the vector of frequency-premiums derived from a Poisson model with regression components depends only on the linear space spanned by the covariates. Hence, the multiplicative coefficients derived from the Poisson model are defined up to a multiplicative constant for each rating factor, whatever are the omitted levels.

- These coefficients can be compared to the relative frequency of each category, which is the frequency of claims for one category divided by the global frequency, column REL. FRE. in Table 1. Consider for instance the category "bulk transport" of the rating factor "firm's activity sector". The relative frequency is 1.617, whereas the standardized coefficient derived from the Poisson model equals 1.146. From the likelihood equations of the Poisson model (see (3)), the number of claims equals the sum of the frequency premiums for each level. The ratio $1.617 / 1.146=1.411$ means that the vehicles belonging to this type of fleet have, with respect to other rating factors, a frequency risk level which is $41 \%$ higher than the average.

Table 1 also provides levels of significance for the coefficients estimated in the regression. The P-VALUE column is obtained from a studentized statistic (i.e. the ratio between the estimated coefficient and its estimated standard 
TABLE 1

RATING SCORE FOR THE FREQUENCY OF CLAIMS WITH BODILY INJURIES

\begin{tabular}{|c|c|c|c|c|}
\hline & WEIGHT (\%) & REL.FRE. & ST.COFF. & P-VALUE \\
\hline \multicolumn{5}{|l|}{ VARIABLE: FIRM'S ACTIVITY SECTOR } \\
\hline general merchandise transport & 13.7 & 1.508 & 1.233 & 0.011 \\
\hline bulk transport & 10.9 & 1.617 & 1.146 & 0.079 \\
\hline short term rental & 2.5 & 0.959 & 0.840 & 0.501 \\
\hline independent trucker, other sector & 72.9 & 0.813 & 0.940 & ref. group \\
\hline \multicolumn{5}{|l|}{ VARIABLE: VeHICLES-YEARS } \\
\hline 0 or 1 vehicle-year & 31.8 & 0.758 & 0.803 & ref. group \\
\hline 2 vehicle-years & 11.9 & 0.887 & 0.920 & 0.145 \\
\hline 3 vehicle-years & 7.2 & 1.032 & 1.055 & 0.010 \\
\hline 4 to 9 vehicle-years & 17.1 & 1.111 & 1.083 & $<0.001$ \\
\hline 10 to 20 vehicle-years & 9.6 & 1.292 & 1.177 & $<0.001$ \\
\hline more than 20 vehicle-years & 22.4 & 1.183 & 1.164 & $<0.001$ \\
\hline \multicolumn{5}{|l|}{ VARIABLE: TYPE OF FUEL } \\
\hline gasoline & 20.4 & 0.430 & 0.597 & $<0.001$ \\
\hline fuel oil & 79.6 & 1.147 & 1.104 & ref. group \\
\hline \multicolumn{5}{|l|}{ VARIABLE: WEIGHT OF THE VEHICLE } \\
\hline from 3,000 to $3,870 \mathrm{kgs}$ & 20 & 0.624 & 0.718 & 0.014 \\
\hline from 3,871 to $6,220 \mathrm{kgs}$ & 20 & 0.674 & 0.888 & 0.025 \\
\hline from 6,221 to $7,620 \mathrm{kgs}$ & 20 & 1.174 & 1.108 & 0.982 \\
\hline from 7,621 to $8,850 \mathrm{kgs}$ & 20 & 1.428 & 1.174 & 0.479 \\
\hline more than $8,850 \mathrm{kgs}$ & 20 & 1.099 & 1.110 & ref. group \\
\hline \multicolumn{5}{|l|}{ VARIABLE: TYPE OF USE } \\
\hline commercial use & 75.8 & 0.809 & 0.969 & 0.508 \\
\hline bulk transport & 10.4 & 1.724 & 1.351 & 0.005 \\
\hline other types of transport & 13.8 & 1.501 & 0.904 & ref. group \\
\hline \multicolumn{5}{|l|}{ VARIABLE: Number OF AXLES } \\
\hline unknown & 1.3 & 5.706 & 6.835 & $<0.001$ \\
\hline 2 axles, less than $4,000 \mathrm{kgs}$ & 21.2 & 0.573 & 1.174 & ref. group \\
\hline 2 axles, more than $4,000 \mathrm{kgs}$ & 26.9 & 0.694 & 0.797 & 0.023 \\
\hline 3 axles & 18.0 & 0.917 & 0.781 & 0.022 \\
\hline 4 axles & 5.4 & 0.908 & 0.760 & 0.028 \\
\hline 5 axles & 8.8 & 0.876 & 0.635 & 0.001 \\
\hline 6 axles and more & 18.4 & 1.775 & 1.141 & 0.869 \\
\hline \multicolumn{5}{|l|}{ VARIABLE: NUMBER OF CYLINDERS } \\
\hline 1 to 5 cylinders & 1.4 & 0.840 & 0.982 & 0.410 \\
\hline 6 to 7 cylinders & 59.9 & 1.261 & 1.122 & $<0.001$ \\
\hline 8 cylinders and more & 38.7 & 0.600 & 0.812 & ref. group \\
\hline Number of vehicles & \multicolumn{4}{|c|}{124,629} \\
\hline
\end{tabular}


deviation). For each rating factor, the reference group is related to the level which was suppressed in order to avoid colinearity.

The frequency of claims increases with the size of the fleet. This result could be explained by a greater exposure to risk (as measured by annual mileage) for the vehicles belonging to large fleets. The same reason probably also explains why gasoline-powered vehicles are much less risky than fuel-powered ones.

Annual mileage was estimated for the vehicles which had a recent mechanical check-up (54,699 vehicles). The estimation of the rating model with this supplementary variable leads to the following results, with a level of significance equal to $10 \%$.

- The fuel effect disappears.

- The size effect decreases, but remains significant.

- The firm activity sectors are not significant.

- The number of cylinders effect disappears.

Detailed results can be obtained in Dionne, Desjardins, Pinquet (1999).

On the sample, the estimators given in the preceding section are equal to

$$
\widehat{V_{R R}}=0.153 ; \widehat{V_{U U}}=1.121 \Rightarrow \widehat{V_{S S}}=\frac{\widehat{V_{U U}}-\widehat{V_{R R}}}{1+\widehat{V_{R R}}}=0.840 \text {. }
$$

The estimated variances of random effects are close to the malus applied to the a priori frequency premium after one claim if this premium is close to zero. This is the case for most of the fleets in the portfolio because of their small size on average, and because of the low frequency of claims for bodily injuries, which is equal to $1.6 \%$ per year on average. Hence, one claim reported on such a fleet would entail a malus close to $15 \%$ for a new vehicle.

The estimated variance $\widehat{V_{S S}}$ of the vehicle-specific random effect is important. The history of a vehicle will have much more ability to predict the risk level of this vehicle than that of the other vehicles in the fleet.

The preceding estimators are not really modified by a "generalized estimating equation" (see the end of Section 3.2). The frequency premiums are very close, and estimated variances of the random effects are

$$
\widehat{V_{R R}}=0.161 ; \widehat{V_{U U}}=1.110 \text {. }
$$

We use the estimators obtained in equation (9) at the end of the section.

Bonus-malus coefficients are computed at the fleet level in Table 2, for the two limit values of the turnover. Credibilities of the histories and standard deviations of the bonus-malus coefficients are given for each size level retained in the regression components (see Table 1).

Since the bonus-malus coefficients are computed at the fleet level, all the averages computed in Table 2 are weighted by the frequency premiums of the fleets. Due to the important value of the variance of the vehicle-specific random effect, the credibility strongly depends on the turnover for fleets with little or 
TABLE 2

AVERAGE CREDIBILITIES FOR FLEETS AND VEHICLES

STANDARD DEVIATIONS OF BONUS-MALUS COEFFICIENTS AT THE FLEET LEVEL

\begin{tabular}{lcccc}
\hline \hline Fleet size & $\overline{\boldsymbol{\alpha}}$ & $\overline{\boldsymbol{\alpha}}+\overline{\boldsymbol{\beta}}$ & $\begin{array}{c}\boldsymbol{\sigma}_{\text {bonmal }} \\
\text { (turnover }=\mathbf{1 0 0 \% )}\end{array}$ & $\begin{array}{c}\boldsymbol{\sigma}_{\text {bonmal }_{\boldsymbol{\alpha}+\boldsymbol{\beta}}} \\
\text { (turnover }=\mathbf{0} \%)\end{array}$ \\
\hline 0 or 1 vehicle-year & 0.003 & 0.019 & 0.020 & $\mathbf{0 . 1 3 6}$ \\
2 vehicle-years & 0.006 & 0.026 & 0.030 & 0.126 \\
3 vehicle-years & 0.009 & 0.030 & 0.037 & 0.122 \\
from 4 to 9 vehicle-years & 0.019 & 0.041 & 0.053 & 0.116 \\
from 10 to 20 vehicle-years & 0.048 & 0.072 & 0.083 & 0.129 \\
more than 20 vehicle-years & 0.245 & 0.262 & 0.189 & 0.203 \\
\hline \hline
\end{tabular}

medium size. The same result holds for the dispersion of the bonus-malus coefficients. As expected from the conclusion of Section 3.1., the standard deviation of the bonus-malus coefficients is not a monotonic function of the size of the fleet when the turnover is equal to zero.

\subsection{An experience rating scheme using full information on the claims history}

Since the drivers do not pay insurance premiums of firm-owned vehicles, the computation of premiums at the vehicle level may appear irrelevant. However, disaggregated information on the premium may be of interest for the firm. In this context, you can think of using full information on the claims history. Different weights can be given to the histories of the vehicles in the derivation of the bonus-malus coefficient for a given vehicle.

Bonus-malus coefficients obtained at the vehicle level from the approach retained in Section 3.3 have a very low within fleets dispersion. This is due to the fact that the credibility granted to the history of the vehicle is applied to a ratio computed at the fleet level. The within fleets dispersion of the bonusmalus coefficients, as measured by the standard deviation, is at most equal to three per cent of the total dispersion for the different size levels.

In this section, we compute linear credibility predictors which give specific weights to the history of each vehicle in the prediction of the risk frequency for a given vehicle. The intuition is that the predictor should overweight the history of this vehicle, as compared to the one obtained in Section 3.3. As a result, the within fleets dispersion of the bonus-malus coefficients should increase.

As in Section 3.3, we consider a fleet with $m$ vehicles during the first period, and a vehicle $i_{0}$ which belongs to the fleet during the forecast period. We suppress the fleet index, and write the bonus-malus coefficient as $\widehat{a_{i_{0}}}+{ }^{t} \widehat{b_{i_{0}}} n$ with

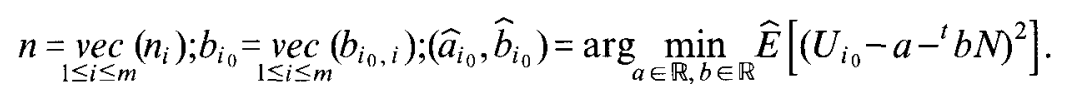


The estimated expectation is derived in the model with random effects. Since $E\left(U_{i_{0}}\right)=1$ the bonus-malus coefficient is equal to

$$
\widehat{a}_{i_{0}}{ }^{t} \widehat{b}_{i_{0}} n=1+{ }^{t} \widehat{b}_{i_{0}}(n-\widehat{E}(N))=1+{ }^{t} \widehat{b}_{i_{0}}(n-\hat{\lambda}) \text {. }
$$

The vector of frequency-premiums $\hat{\lambda}=v e c_{1 \leq i \leq m}\left(\widehat{\lambda_{i}}\right)$ is derived from m.l.e. in the a priori rating model. It is a consistent estimator for the frequency risks in the model with random effects. From the consistent estimators of individual variances and covariances derived in Section 3.3, we infer

$$
\widehat{b}_{i_{0}}=[\widehat{V}(N)]^{-1} \widehat{\operatorname{Cov}}\left(N, U_{i_{0}}\right),
$$

with

$$
\begin{gathered}
\hat{V}(N)=D+\left(\widehat{V_{R R}} \hat{\lambda}^{t} \widehat{\lambda}\right), D=\operatorname{diag}\left(\widehat{\lambda_{i}}+\left[\widehat{\lambda_{i}^{2}}\left(\widehat{V_{U U}}-\widehat{V_{R R}}\right)\right]\right) ; \\
\widehat{\operatorname{Cov}}\left(N, U_{i_{0}}\right)=\widehat{V_{R R}} \widehat{\lambda}+\left(\widehat{V_{U U}}-\widehat{V_{R R}}\right) \widehat{\lambda_{i_{0}}} e_{i_{0}} .
\end{gathered}
$$

The last term exists if $i_{0} \leq m$ (i.e. the vehicle was observed during the first period). The vector $e_{i_{0}}$ belongs to the canonical basis of $\mathbb{R}^{m}$, with the corresponding index.

Let us compute $[\widehat{V}(N)]^{-1}$ : From $\widehat{V}(N)=D\left[I_{m}+\left(\widehat{V_{R R}} D^{-1} \hat{\lambda}^{t} \widehat{\lambda}\right)\right]$ and $\left(D^{-1} \hat{\lambda}^{t} \hat{\lambda}\right)^{2}=$ $\|\widehat{\lambda}\|_{D^{-1}}^{2} D^{-1} \hat{\lambda}^{t} \hat{\lambda},\|\hat{\lambda}\|_{D^{-1}}^{2}={ }^{t} \hat{\lambda} D^{-1} \hat{\lambda}=\sum_{i=1}^{m} \frac{\widehat{\lambda_{i}}}{1+\left[\lambda_{i}\left(\widehat{V_{U U}}-\widehat{V_{R R}}\right)\right.}$,

we obtain

$$
[\widehat{V}(N)]^{-1}=\left[I_{m}+\left(\widehat{V_{R R}} D^{-1} \widehat{\lambda}^{t} \widehat{\lambda}\right)\right]^{-1} D^{-1}=\left[I_{m}-\left(\frac{\widehat{V_{R R}}}{1+\| \widehat{\lambda}_{D^{-1}}^{2} \widehat{V_{R R}}} D^{-1} \widehat{\lambda} t \widehat{\lambda}\right)\right] D^{-1}
$$

A first expression of $\widehat{b}_{i_{0}}=[\widehat{V}(N)]^{-1} \widehat{\operatorname{Cov}}\left(N, U_{i_{0}}\right)$ is

$$
\widehat{b}_{i_{0}}=\left[I_{m}-\left(\frac{\widehat{V_{R R}}}{1+\|\widehat{\lambda}\|_{D^{-1}}^{2} \widehat{V_{R R}}} D^{-1} \widehat{\lambda}^{i} \widehat{\lambda}\right)\right] D^{-1}\left[\widehat{V_{R R}} \widehat{\lambda}+\left(\widehat{V_{U U}}-\widehat{V_{R R}}\right) \widehat{\lambda e_{i_{0}}}\right]
$$

Since

$$
\left[I_{m}-\left(\frac{\widehat{V_{R R}}}{1+\| \widehat{\lambda \|}_{D^{-1}}^{2} \widehat{V_{R R}}} D^{-1} \widehat{\lambda}^{t} \hat{\lambda}\right)\right] D^{-1} \hat{\lambda}=\frac{1}{1+\|\widehat{\lambda}\|_{D^{-1}}^{2} \widehat{V_{R R}}} D^{-1} \hat{\lambda}
$$


we have

$$
\widehat{b}_{i_{0}}=\frac{1}{1+\| \widehat{\lambda \|_{D^{-1}}^{2} \widehat{V_{R R}}}}\left[\left(\widehat{V_{R R}} D^{-1} \widehat{\lambda}\right)+\widehat{\lambda_{i_{0}}}\left(\widehat{V_{U U}}-\widehat{V_{R R}}\right) D^{-1} e_{i_{0}}\right]
$$

The bonus-malus coefficient for the vehicle $i_{0}$ is obtained from the credibility formula

$$
1+\widehat{b}_{i_{0}}(n-\hat{\lambda})=\left[1+\sum_{i=1}^{m} a_{i}\left(\frac{n_{i}}{\widehat{\lambda}_{i}}-1\right)\right]+\widehat{\beta}_{i_{0}}\left(\frac{n_{i_{0}}}{\widehat{\lambda}_{i_{0}}}-1\right)
$$

if we write

$$
a_{i}=\hat{\lambda}_{i} \hat{b}_{i_{0}, i}\left(i \neq i_{0}\right) ; a_{i_{0}}+\beta_{i_{0}}=\hat{\lambda}_{i_{0}} \hat{b}_{i_{0}, i_{0}}
$$

with $a_{i_{0}}$ expressed as the $\alpha_{i}, i \neq i_{0}$. The bonus-malus coefficient is a sum of two terms:

- The first one does not depend on the vehicles within the fleet, and is applied to the new vehicles.

- The second one exists only if the vehicle was observed in the past $\left(1 \leq i_{0} \leq m\right)$. The credibility coefficient $\beta_{i_{0}}$ is applied to the individual history. The corresponding coefficient was applied to the history at the fleet level in Section 3.3, and this explains the more important within fleet dispersion of bonus-malus coefficients which use all the information.

From equations (10) and (11), the credibility coefficients are respectively equal to

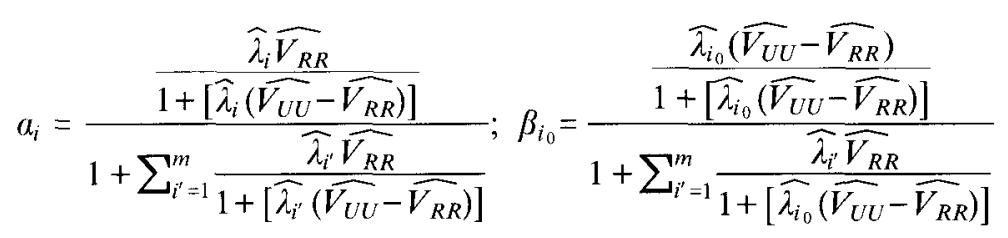

$\forall i, i_{0}=1, \ldots, m$. As in Section 3.3, this credibility system makes sense only if $\widehat{V_{U U}}>\widehat{V_{R R}}$ which means that the estimated variance $\widehat{V_{S S}}$ of the vehicle-specific effect is greater than 0 (see equation (5)).

Let us compare results obtained in this section and in Section 3.3 with an example. We use the estimations given in equation (9). Consider a fleet of five vehicles observed during a period, with frequency-premiums equal to 0.02 for each vehicle. Suppose that one claim was reported during the first period. The bonus-malus coefficients for the next period are given in the following table. 
TABLE 3

BONUS-MALUS COEFFICIENTS WITH LIMITED AND FULL INFORMATION (EXAMPLE)

\begin{tabular}{lcc}
\hline \hline Fleet size & History at the fleet level & Full information \\
\hline new vehicle & 1.133 & 1.135 \\
claimless vehicle & 1.301 & 1.116 \\
vehicle with one claim reported & 1.301 & 2.063 \\
\hline \hline
\end{tabular}

Let us comment the coefficients given by the BMS with full information. The four vehicles without claim reported are penalized because the malus at the fleet level outweighs the bonus generated by the individual history. The bonus-malus coefficient of the vehicle with one claim reported is much more important than that of the four other ones because of the differences between the individual credibilities.

The within fleets dispersion of bonus-malus coefficients will be more important with this BMS. This is shown in the following table which provides between fleets and total standard deviation of bonus-malus coefficients computed on the portfolio with the present BMS.

TABLE 4

TOTAL AND BETWEEN FLEETS STANDARD DEVIATIONS OF BONUS-MALUS COEFFICIENTS LINEAR CREDIBILITY WITH FULL INFORMATION

\begin{tabular}{lccc}
\hline \hline Fleet size & $\begin{array}{c}\text { turnover }=\mathbf{1 0 0} \% \\
\boldsymbol{\sigma}_{\text {between }}\end{array}$ & $\begin{array}{c}\text { turnover }=\mathbf{0 \%} \\
\boldsymbol{\sigma}_{\text {between }}\end{array}$ & $\begin{array}{c}\text { turnover }=\mathbf{0} \% \\
\boldsymbol{\sigma}_{\text {total }}\end{array}$ \\
\hline 0 or 1 vehicle-year & 0.020 & 0.136 & 0.138 \\
2 vehicle-years & 0.030 & 0.123 & 0.151 \\
3 vehicle-years & 0.037 & 0.123 & 0.158 \\
from 4 to 9 vehicle-years & 0.053 & 0.116 & 0.169 \\
from 10 to 20 vehicle-years & 0.083 & 0.128 & 0.187 \\
more than 20 vehicle-years & 0.189 & 0.203 & 0.231 \\
\hline \hline
\end{tabular}

The between fleets dispersions of the bonus-malus coefficients are very close to those obtained in Table 2 for the same value of the turnover. This means that using only the history of the fleet in the prediction did not entail a loss of efficiency for bonus-malus coefficients computed at the fleet level.

Optimal BMS using all the information on the claim history can also be derived with an expected value principle (Lemaire (1985), Dionne et al. (1989), Pinquet (1997)). The negative binomial model with random effects (Hausman, Hall, Griliches (1984)) can be used for that purpose. Initially designed for longitudinal count data, it can be used in our context, due to the analogy between panel data and stratified samples. For example, consider an individual as a 
stratum and a period as an individual within a stratum. This model is developed in Dionne, Desjardins, Pinquet (2000a).

\section{Bonus-malus Systems From the NUMBeR OF SAFETY OFFENCES}

\subsection{Safety offences used as regression components}

Owing to the no-fault setting, the history of claims cannot be included in the tariff structure of the SAAQ. However, safety offences can be used to perform experience rating. In our data base, safety offences of different types were recorded at the carrier level and at the driver level. Those which were recorded in 1995 are added here as regression components in the Poisson model estimated in Table 1. Hence the number of claims reported in 1996 is explained by rating factors and by the safety offences recorded the year before. Each estimated coefficient related to a given type of safety offence leads to a relative malus, if this coefficient is positive. The safety offences which did entail a malus are presented in Table 5 .

TABLE 5

RELATIVE MALI DERIVED FROM SAFETY OFFENCES

\begin{tabular}{lccc}
\hline \hline $\begin{array}{l}\text { Type of safety offence } \\
\text { recorded in 1995 }\end{array}$ & related to & $\begin{array}{c}\text { relative } \\
\text { malus (\%) }\end{array}$ & P-value \\
\hline exceeding speed limits & vehicles & 42 & $<0.001$ \\
\hline not wearing the seat belt & vehicles & 93 & $<0.001$ \\
not respecting hazardous goods rules & carriers & 105 & 0.008 \\
excess load & carriers & 12 & 0.089 \\
not stopping at an agent's signal & vehicles & 38 & 0.091 \\
not respecting driving hours rules & carriers & 72 & 0.013 \\
\hline number of vehicles & & 100,048 & \\
\hline \hline
\end{tabular}

We retained the vehicles with a positive duration of authorization for the licence plate during 1995 and 1996. Other safety offences which were not retained by the model are the following: Exceeding size limits, not respecting bulk trucking regulation, not respecting mechanical check-up rules, driving with a sanction, not stopping at a red light. Many of them are significant when we consider all types of road accidents (property damages and bodily injuries). See Dionne et al. (1999) for more details, including regression results related to the rating factors. An optimal BMS is designed in the next section from a model with random effects on two types of events, namely the claims for bodily injury and all the safety offences. 


\subsection{The model with random effects}

Let $I N F_{f i}$ be the number of safety offences (whatever their type) recorded on the vehicle $i$ belonging to the fleet $f$. We write

$$
I N F_{f i} \sim P\left(\tau_{f i} t_{f i}\right)
$$

where $\tau_{f i}=d_{f i} \exp \left(x_{f} \zeta+z_{f i} \eta\right)$ is the component of $E\left(I N F_{f i}\right)$ which is explained by the duration of exposure to safety offences and by both fleet-specific and vehicle-specific regression components, and where $t_{f i}$ is the fixed effect. The hierarchical structure of the portfolio is taken into account by writing $t_{f i}=p_{f} q_{f i}$ where $p_{f}$ and $q_{f i}$ are the fleet-specific and vehicle-specific fixed effects. All the number variables are supposed independent in the fixed effects model. Let $U$, $R, T$ and $P$ be random variables with the same joint distribution as any random vector such as $\left(U_{f i}, R_{f}, T_{f i}, P_{f}\right)$ (we use the notations of Section 3.2). The assumption $E(U)=1$ made in Section 3.2 is relaxed now. A natural multivariate distribution family with explicit moments for non-negative random effects is the log-normal distribution family, and the expectation depends then on the variance. Let $\widehat{\tau_{f i}}=d_{f i} \exp \left(x_{f} \widehat{\zeta}+z_{f i} \widehat{\eta}\right)$ be the estimation of $E\left(I N F_{f i}\right)$ derived from likelihood maximization in the Poisson model without fixed or random effects. If data are generated in the random effects model, we have

$$
\widehat{\lambda_{f i}} \rightarrow E\left(N_{f i}\right)=\lambda_{f i} E(U) ; \widehat{\tau_{f i}} \rightarrow E\left(I N F_{f i}\right)=\tau_{f i} E(T) \text {. }
$$

The expectation is computed in the random effects model. From (12) and results similar to those given in (2), we obtain the following limits

$$
\begin{aligned}
& \widehat{V_{U T}}=\frac{\sum_{f, i}\left(N_{f i}-\widehat{\lambda_{f i}}\right)\left(I N F_{f i}-\widehat{\tau_{f i}}\right)}{\sum_{f, i} \widehat{\lambda_{f i}} \widehat{\tau_{f i}}} \rightarrow \frac{\operatorname{Cov}(U, T)}{E(U) E(T)} \\
& \widehat{V_{R P}}=\frac{\sum_{f} \sum_{1 \leq i, i^{\prime} \leq m_{f} ; i \neq i^{\prime}}\left(N_{f i}-\widehat{\lambda_{f i}}\right)\left(I N F_{f i}-\widehat{\tau_{f i}}\right)}{\sum_{f} \sum_{1 \leq i, i^{\prime} \leq m_{f} ; i \neq i^{\prime}} \widehat{\lambda_{f i}} \widehat{\tau_{f f}}} \rightarrow \frac{\operatorname{Cov}(R, P)}{E(R) E(P)} \\
& \widehat{V_{P P}}=\frac{\sum_{f} \sum_{1 \leq i, i^{\prime} \leq m_{f} ; i \neq i^{\prime}}\left(I N F_{f i}-\widehat{\tau_{f i}}\right)\left(I N F_{f i}-\widehat{\tau_{f t}}\right)}{\sum_{f 1 \leq i, i^{\prime} \leq m_{f} ; i \neq i^{\prime}} \sum_{\widehat{\tau_{f i}} \widehat{\tau_{f t}}}} \rightarrow C V^{2}(P)=\frac{V(P)}{E^{2}(P)} ; \\
& {\widehat{V_{T T}}}^{1}=\frac{\sum_{f, i}\left[\left(I N F_{f i}-\widehat{\tau_{f i}}\right)^{2}-\widehat{\tau_{f i}}\right]}{\sum_{f, i}{\widehat{\tau_{f i}}}^{2}} \rightarrow C V^{2}(T)=\frac{V(T)}{E^{2}(T)} \text {. }
\end{aligned}
$$


The superscript " 1 " is used for the preceding estimators because they are obtained at the first step of the Newton-Raphson algorithm of likelihood maximization, where the initial value is the m.l.e. for the a priori rating model. For instance, the estimator $\widehat{V_{R P}} 1$ reflects the predictive power that safety offences recorded on a given vehicle have on the risk level of every other vehicle in the same fleet. Not surprisingly, the fleet-specific credibility obtained in the next section will depend on this estimator.

\subsection{Linear credibility predictors}

An optimal BMS using both claims and safety offences would be more efficient than those designed in the preceding sections (see Pinquet (1998) for a comparison of short-term effects). We now consider the case where claims cannot be used and the frequency of claims is predicted from the history of safety violations only. Notice that an optimal BMS using both claims and safety offences could easily be obtained from the preceding estimators. It would be enough to adapt the linear credibility system given in the aforementioned paper to a stratified portfolio.

Let us compute the bonus-malus coefficient for the frequency of claims reported by the vehicle $i_{0}$ belonging to the fleet $f_{0}$. The fleet index is suppressed in order to simplify the expressions. The bonus-malus coefficient is written a $\widehat{a_{i 0}}+\widehat{b_{i_{0}}}\left(\sum_{i=1}^{m} \mathrm{inf}_{i}\right)$, with

$$
\left(\widehat{a_{i_{0}}}, \widehat{b_{i_{0}}}\right)=\arg \min _{a, b} \widehat{E}\left[\left(\frac{U_{i_{0}}}{E\left(U_{i_{0}}\right)}-a-b\left(\sum_{i=1}^{m} I N F_{i}\right)\right)^{2}\right] .
$$

From computations similar to those performed in Section 3.3, we obtain the following bonus-malus coefficient

$$
\text { bonmal }_{i_{0}}=1+\widehat{b_{i_{0}}}\left(\sum_{i=1}^{m}\left(\inf _{i}-\widehat{\tau_{i}}\right)\right) ; \widehat{b_{i_{0}}}=\frac{\widehat{\operatorname{Cov}}\left(\frac{U_{i_{0}}}{E\left(U_{i_{0}}\right)}, \sum_{i=1}^{m} I N F_{i}\right)}{\widehat{V}\left(\sum_{i=1}^{m} I N F_{i}\right)},
$$

with

$$
\widehat{\operatorname{Cov}}\left(\frac{U_{i_{0}}}{E\left(U_{i_{0}}\right)}, \sum_{i=1}^{m} I N F_{i}\right)={\widehat{V_{R P}}}^{1}\left(\sum_{i=1}^{m} \widehat{\tau_{i}}\right)+\left(\left({\widehat{V_{U T}}}^{1}-{\widehat{V_{R P}}}^{1}\right){\widehat{\tau_{i 0}}}^{\prime}\right)
$$


The last term must be suppressed if the vehicle $i_{0}$ is not observed during the first period. Following the computations of Section 3.3, we obtain then

$$
\begin{aligned}
& \widehat{V}\left(\sum_{i=1}^{m} I N F_{i}\right)=\left(\sum_{i=1}^{m} \widehat{\tau}_{i}\right)+\left(\widehat{V} P P^{1}\left(\sum_{i=1}^{m}{\widehat{\tau_{i}}}^{2}\right)^{2}\right)+\left[\left({\widehat{V_{T T}}}^{1}-{\widehat{V_{P P}}}^{1}\right)\left(\sum_{i=1}^{m} \widehat{\tau}_{i}^{2}\right)\right] ; \\
& \text { bonmal }_{i_{0}}=\left(1-\operatorname{cred}_{i_{0}}\right)+\operatorname{cred}_{i_{0}} \frac{\sum_{i=1}^{m} \inf _{i}}{\sum_{i=1}^{m} \widehat{\tau_{i}}} ; \\
& \operatorname{cred}_{i_{0}}=\alpha\left(i_{0} \notin\{1, \ldots, m\}\right) ; \operatorname{cred}_{i_{0}}=\alpha+\beta_{i_{0}}\left(i_{0} \in\{1, \ldots, m\}\right) ; \\
& a=\frac{\widehat{V_{R P}}\left(\sum_{i=1}^{m}{\widehat{\tau_{i}}}^{-}\right.}{1+\left(\widehat{V_{P P}}\left(\sum_{i=1}^{m} \widehat{\tau}_{i}\right)\right)+\left[\left(\widehat{V_{T P}}-\widehat{V_{P P}}\right)\left(\frac{\sum_{i=1}^{m} \widehat{\tau}_{i}^{2}}{\sum_{i=1}^{m} \widehat{\tau}_{i}}\right)\right]} \\
& \beta_{i_{0}}=\frac{\left(\widehat{V_{U T}}-\widehat{V_{R P}}\right) \widehat{\tau_{i_{0}}}}{1+\left(\widehat{V_{P P}}\left(\sum_{i=1}^{m} \widehat{\tau_{i}}\right)\right)+\left[\left(\widehat{V_{T T}}{ }^{1}-\widehat{V}_{P P}^{1}\right)\left(\frac{\sum_{i=1}^{m} \widehat{\tau}_{i}^{2}}{\sum_{i=1}^{m} \widehat{\tau}_{i}}\right)\right]} .
\end{aligned}
$$

The fleet-specific credibility coefficient $a$ increases with ${\widehat{V_{R P}}}^{1}$ a term related to the covariance between the two fleet-specific random effects. The coefficient $\beta_{i_{0}}$ is the vehicle-specific credibility. It makes sense only if ${\widehat{V_{U T}}}^{1}>{\widehat{V_{R P}}}^{1}$, a condition fulfilled in our data.

\subsection{Empirical results}

The frequency of claims with bodily injury reported in 1996 is predicted from the number of safety offences recorded in 1995, and we retained the vehicles with a positive duration of authorization for the licence plate during 1995 and 1996. The detailed results of the regression explaining the number of safety offences recorded in 1995 are presented in Table 6. Let us emphasize two points:

- The annual frequency of recorded offences is equal to $22.2 \%$. It is much superior to that of the claims with bodily injury liability. This will explain later the better short term performance of the prediction designed in this section.

- The frequency of offences increases with the size of the fleet, but decreases for fleets with more than 20 vehicle-years. 
TABLE 6

RATING SCORE FOR THE FREQUENCY OF SAFETY OFFENCES

\begin{tabular}{|c|c|c|c|c|}
\hline & WEIGHT $(\%)$ & REL.FRE. & ST.COFF. & P-VALUE \\
\hline \multicolumn{5}{|l|}{ VARIABLE: FIRM'S ACTIVITY SECTOR } \\
\hline general merchandise transport & 13.3 & 1.269 & 1.048 & 0.013 \\
\hline bulk transport & 10.7 & 2.045 & 0.997 & 0.314 \\
\hline short term rental & 2.5 & 1.297 & 1.742 & $<0.001$ \\
\hline independent trucker, other sector & 73.5 & 0.789 & 0.967 & ref. group \\
\hline \multicolumn{5}{|l|}{ VARIABLE: VEHICLES-YEARS } \\
\hline 0 or 1 vehicle-year & 32.7 & 0.953 & 1.055 & ref. group \\
\hline 2 vehicle-years & 11.4 & 1.022 & 1.119 & 0.008 \\
\hline 3 vehicle-years & 7.1 & 1.147 & 1.174 & $<0.001$ \\
\hline 4 to 9 vehicle-years & 17.4 & 1.262 & 1.210 & $<0.001$ \\
\hline 10 to 20 vehicle-years & 9.7 & 1.256 & 1.056 & 0.725 \\
\hline more than 20 vehicle-years & 21.7 & 0.686 & 0.606 & $<0.001$ \\
\hline \multicolumn{5}{|l|}{ VARIABLE: TYPE OF FUEL } \\
\hline gasoline & 22.2 & 0.392 & 0.582 & $<0.001$ \\
\hline fuel oil & 77.8 & 1.174 & 1.119 & ref. group \\
\hline \multicolumn{5}{|l|}{ VARIABLE: WEIGHT OF THE VEHICLE } \\
\hline from 3,000 to $3,870 \mathrm{kgs}$ & 20.0 & 0.653 & 0.991 & 0.371 \\
\hline from 3,871 to $6,220 \mathrm{kgs}$ & 20.0 & 0.654 & 0.939 & $<0.001$ \\
\hline from 6,221 to $7,620 \mathrm{kgs}$ & 20.5 & 1.112 & 0.960 & $<0.001$ \\
\hline from 7,621 to $8,850 \mathrm{kgs}$ & 19.2 & 1.517 & 1.061 & 0.473 \\
\hline more than $8,850 \mathrm{kgs}$ & 20.3 & 1.082 & 1.050 & ref. group \\
\hline \multicolumn{5}{|l|}{ VARIABLE: TYPE OF USE } \\
\hline commercial use & 76.0 & 0.776 & 0.927 & 0.644 \\
\hline bulk transport & 10.2 & 2.356 & 1.667 & $<0.001$ \\
\hline \multicolumn{5}{|l|}{ VARIABLE: NUMBER OF AXLES } \\
\hline unknown & 2.3 & 0.998 & 1.119 & 0.047 \\
\hline 2 axles, less than $4,000 \mathrm{kgs}$ & 20.6 & 0.651 & 0.984 & ref. group \\
\hline 2 axles, more than $4,000 \mathrm{kgs}$ & 27.6 & 0.600 & 0.751 & $<0.001$ \\
\hline 3 axles & 18.3 & 0.802 & 0.696 & $<0.001$ \\
\hline 4 axles & 5.7 & 0.893 & 0.856 & 0.017 \\
\hline 5 axles & 8.2 & 0.987 & 0.865 & 0.044 \\
\hline 6 axles and more & 17.3 & 2.305 & 1.836 & $<0.001$ \\
\hline \multicolumn{5}{|l|}{ VARIABLE: NUMBER OF CYLINDERS } \\
\hline 1 to 5 cylinders & 1.4 & 0.714 & 0.834 & 0.699 \\
\hline 6 to 7 cylinders & 59.0 & 1.294 & 1.130 & $<0.001$ \\
\hline 8 cylinders and more & 39.6 & 0.572 & 0.812 & ref. group \\
\hline Number of vehicles & \multicolumn{4}{|c|}{100,048} \\
\hline
\end{tabular}


As for the random effects, the numerical values of the estimators are

$$
{\widehat{V_{U T}}}^{1}=0.519 ;{\widehat{V_{P P}}}^{1}=0.465 ;{\widehat{V_{R P}}}^{1}=0.141 ;{\widehat{V_{T T}}}^{1}=1.263 \text {. }
$$

These moment-based estimators can be connected to explicit distributions. If log-normal distributions are retained for the random effects, we can write

$$
R=\exp \left(a_{1} G_{1}\right) ; U_{1}=\exp \left(a_{1} G_{1}+a_{2} G_{2}^{i}\right) \Rightarrow U_{i}=R S_{i}, S_{i}=\exp \left(a_{2} G_{2}^{i}\right) .
$$

The fleet index is suppressed, and the random variables $G_{1},\left(G_{2}^{i}\right)_{i=1, \ldots, m}$ follow independent standard normal distributions. In the same way, we can write

$$
\begin{gathered}
P=\exp \left(a_{3} G_{1}+a_{4} G_{3}\right) ; T_{i}=\exp \left(a_{3} G_{1}+a_{4} G_{3}+a_{5} G_{2}^{i}+a_{6} G_{4}^{i}\right) \Rightarrow T_{i}=P Q_{i}, \\
Q_{i}=\exp \left(a_{5} G_{2}^{i}+a_{6} G_{4}^{i}\right),
\end{gathered}
$$

with similar assumptions on the random variables $G_{3},\left(G_{4}^{i}\right)_{i=1, \ldots, m}$. It is easily seen that

$$
G \sim N\left(0, I_{q}\right) \Rightarrow \frac{\operatorname{Cov}\left({ }^{t} a G, b G\right)}{E\left({ }^{t} a G\right) E\left({ }^{t} b G\right)}=\exp \left({ }^{t} a b\right)-1
$$

$\forall \mathrm{a}, \mathrm{b} \in \mathbb{R}^{q}$. The moment-based estimators are then connected with the following values

$$
a_{1}=0.381 ; a_{2}=0.828 ; a_{3}=0.346 ; a_{4}=0.512 ; a_{5}=0.346 ; a_{6}=0.562 .
$$

The predictor computed in this section cannot be consistent with respect to the fleet specific component, since the event for which the frequency is predicted is not retained in the history. When the size of the fleet $m$ converges towards infinity, we obtain from equation (14)

$$
\lim _{m \rightarrow+\infty} \operatorname{cred}_{i_{0}}=\frac{\widehat{V_{R P}} 1}{\widehat{V_{P P}} 1}=0.303 \forall i_{0} .
$$

The credibility coefficient cred $_{i_{0}}$ is defined in (14). As we have the following limit

$$
\lim _{m \rightarrow+\infty} \frac{\sum_{i=1}^{m} I N F_{i}}{\sum_{i=1}^{m} \widehat{\tau_{i}}}=\frac{P}{E(P)}
$$

in the random effects model, the limit of the bonus-malus coefficient is

$$
\lim _{m \rightarrow+\infty} \text { bonmal }_{i_{0}}=\left(1-\frac{\widehat{V_{R P}}}{\widehat{V_{P P}}}\right)+\left(\frac{\widehat{V_{R P}}}{\widehat{V_{P P}}} \frac{P}{E(P)}\right) .
$$


Hence, the BMS is not consistent (the limit should be $R / E(R)$ for a consistent predictor). The limit is the estimated affine regression of $R / E(R)$ with respect to $P / E(P)$.

Although this BMS is less efficient in the long run than the one based on the number of claims, it is more efficient after one year, as shown in Table 7.

TABLE 7

AVERAGE CREDIBILITIES FOR FLEETS AND VEHICLES STANDARD DEVIATIONS OF BONUS-MALUS COEFFICIENTS AT THE FLEET LEVEL

\begin{tabular}{lcccc}
\hline \hline Fleet size & $\overline{\boldsymbol{\alpha}}$ & $\overline{\boldsymbol{\alpha}}+\overline{\boldsymbol{\beta}}$ & $\boldsymbol{\sigma}_{\text {bonmal }_{\boldsymbol{\alpha}}}$ & $\boldsymbol{\sigma}_{\text {bonmal }_{\boldsymbol{u}+\beta}}$ \\
\hline 0 or 1 vehicle-year & 0.027 & 0.094 & 0.064 & 0.216 \\
2 vehicle-years & 0.049 & 0.113 & 0.087 & 0.198 \\
3 vehicle-years & 0.070 & 0.132 & 0.107 & 0.196 \\
from 4 to 9 vehicle-years & 0.114 & 0.168 & 0.136 & 0.197 \\
from 10 to 20 vehicle-years & 0.175 & 0.209 & 0.186 & 0.222 \\
more than 20 vehicle-years & 0.242 & 0.252 & 0.220 & 0.235 \\
\hline \hline
\end{tabular}

Table 7 was obtained in the same way as Table 2. Standard deviations of bonusmalus coefficients are more important in this table for fleets with little or medium size. This BMS is less efficient in the long run than the one presented in Section 3, but it is closer to its limit, due to the higher frequency of safety offences.

\section{CONCLUSION}

The objective of this paper was to propose BMS for fleets of vehicles. The models were applied to fleets of trucks, but they could be used for other stratified portfolios if individual information on the insurance contracts was available.

Two systems were presented: one based on past accidents and the other based on past safety offenses. It was shown that the former system is more efficient in the long run, while it is outperformed by the latter BMS after one year, a result explained by the higher frequency of safety offences.

Many extensions of this article can be done. One is to use information on many periods in order to build up a panel. The corresponding panel would be very useful to analyze the stability of the BMS over time. It would also permit to verify for how long period the system based on safety offences dominates the one based on accidents. However such extensions will not be straightforward since we would have to introduce dynamic random effects along with the fleet effects in order to take into account the serial correlations. 


\section{REFERENCES}

1. BOYER, M. and DiONNE, G. (1987) Description and analysis of the quebec automobile insurance plan. Canadian Public Policy 13, 181-195.

2. BÜHLMANN, H. (1967) Experience rating and credibility. ASTIN Bulletin 4, 199-207.

3. Devlin, R.A. (1992) Liability versus no-fault automobile insurance regimes: An analysis of the experience in Quebec. Contributions to Insurance Economics (Editor: Georges Dionne), Kluwer Academic Publishers. Huebner International Series on Risk, Insurance, and Economic Security, Boston.

4. DionNe, G., and VANASSE, C. (1989) A generalization of automobile insurance rating models: The negative binomial distribution with a regression component. ASTIN Bulletin 19, 199212.

5. Dionne, G., and Vanasse, C. (1997a) Une evaluation empirique de la nouvelle tarification de l'assurance automobile au Québec. Actualité Economique 73, 47-80.

6. Dionne, G., Gagné, R., Gagnon, F. and Vanasse, C. (1997b) Debt, moral hazard and airline safety: An empirical evidence. Journal of Econometrics 79, 379-402 (Special Issue on Duration, Transition and Count Data Models. Editors: Christian Gouriéroux and Thierry Magnac).

7. Dionne, G., Desjardins, D. and Pinquet, J. (1999) L'évaluation du risque d'accident des transporteurs en fonction de leur secteur d'activité, de la taille de leur flotte et de leur dossier d'infractions. Research Report 99-28, Centre de Recherches sur les Transports, Université de Montréal.

8. Dionne, G., Desjardins, D. and Pinquet, J. (2000a) Optimal insurance for fleets of vehicles under asymmetric information. Mimeo, Risk Management Chair, HEC Montreal.

9. Dionne, G., Maurice, M. and Pinquet, J. (2000b) The role of memory and saving in longterm contracting with moral hazard: An empirical evidence in automobile insurance. Mimeo, Risk Management Chair, HEC-Montreal.

10. Gouriéroux, C., Monfort, A. and Trognon, A. (1984) Pseudo likelihood methods: Applications to Poisson models. Econometrica 52, 701-720.

11. Hausman, J.A., Hall, B.H. and Griliches, Z. (1984) Econometric models for count data with an application to the patents - R\&D relationship. Econometrica 52, 909-938.

12. JewELL, W.S. (1975) The use of collateral data in credibility theory: A hierarchical model. Giornale dell'Instituto degli Attuari 38, 1-16 (quoted in "Advanced Risk Theory," F. Etienne de Vylder, Edition de l'Université de Bruxelles et Swiss Association of Actuaries, (1996)).

13. Lemaire, J. (1985) Automobile Insurance: Actuarial Models. Huebner International Series on Risk, Insurance and Economic Security, Kluwer Academic Publishers, Boston.

14. LIANG, K.Y., and ZEGER, S.L. (1986) Longitudinal data analysis using generalized linear models. Biometrika 73, 13-22.

15. Marie-Jéanne, P. (1994) Problèmes spécifiques des flottes automobiles. Proceedings of the ISUP conference "Cours Avancé sur l'Assurance Automobile".

16. PINQUET, J. (1997) Allowance for cost of claims in bonus-malus systems. ASTIN Bulletin 27, No. 1, 33-57.

17. PinqueT, J. (1998) Designing optimal bonus-malus systems from different types of claims. ASTIN Bulletin 28, No. 2, 205-220.

18. PINQUeT, J. (2000) Experience rating through heterogeneous models. Handbook of Insurance, chapter 14 (Editor: Georges Dionne), Kluwer Academic Publishers. Huebner International Series on Risk, Insurance and Economic Security, 459-500.

19. Teugels, J.L. and SundT, B. (1991). "A stop-loss experience rating scheme for fleets of cars". Insurance: Mathematics and Economics, North-Holland, 173-179.

\section{Denise Desiardins \\ Centre de Recherche sur les Transports \\ Université de Montréal \\ C.P. 6128, Succursale Centre-ville}


Montréal (Québec)

Canada $\mathrm{H3C} 3 \mathrm{~J} 7$

email:denise@crt.umontreal.ca

Georges Dionne

Ecole des Hautes Etudes Commerciales

Chaire de Gestion des Risques

3000, chemin de la Côte-Sainte-Catherine

Montréal (Québec)

Canada H3T $2 A 7$

email: georges.dionne@hec.ca

Jean Pinquet

U.F.R. de Sciences Economiques

Université de Paris $X$

200, avenue de la République

92001 Nanterre Cedex

France

email:pinquet@u-paris10.fr 